\title{
Effects of co-vibrotactile stimulations around the torso on non-volitional postural responses
}

\author{
Beom-Chan Lee, Member, IEEE, Allison Ho, Bernard J. Martin, \\ and Kathleen H. Sienko, Member, IEEE
}

\begin{abstract}
The purpose of this study was to characterize the effects of co-vibrotactile stimulations around the torso on non-volitional postural responses in the absence of instructions. Four healthy young adults maintained an upright, erect posture with their eyes closed in two different stance conditions: normal and Romberg stance. Six vibrotactile transducers (tactors) were placed on the skin over the right and left external oblique, internal oblique, and erector spinae muscles. Either a combination of vibration at two locations or all locations around the torso was applied for $5 \mathrm{~s}$ during each experimental trial. Regardless of stance condition, vibration applied concurrently over the right and left internal oblique muscle locations and the right and left erector spinae muscle locations induced a postural shift in the anterior and posterior directions, respectively. For these two stimulation conditions, the root-mean-square of sway in the anterior-posterior direction was significantly greater during vibration than before or after stimulation. However, simultaneous activation of all tactors, a combination of right internal oblique and right erector spinae locations, and a combination of left internal oblique and left erector spinae locations did not produce significant directional postural shifts or increases in sway, regardless of the stance condition. These findings suggest that stimuli combinations contribute to a vector summation of individual postural responses described in our previous work and that they could be leveraged in balance-related applications of sensory augmentation vibrotactile displays.
\end{abstract}

\section{INTRODUCTION}

$\mathrm{S}_{\mathrm{u}}^{\mathrm{n}}$ NCE 2001, vibrotactile biofeedback displays have been used as a form of real-time sensory augmentation for balance-related applications. In general, the displays map directions of postural sway captured by a sensor with vibrating actuators around the torso. These "alarm signals" allow users to correct postural sway that would compromise balance [1-8]. When the body tilt exceeds the pre-defined threshold, one of the tactors vibrates to indicate the direction of body correction. Wall et al. [9] initially selected repulsive ("move away from the vibration") versus attractive ("move toward the vibration") cuing based on the notion that such

This work was supported by the National Science Foundation's CAREER program (RAPD-0846471, funded under the American Recovery and Reinvestment Act of 2009).

B. C. Lee is with the Dept. of Mechanical Engineering, University of Michigan, Ann Arbor, MI 48109 USA (e-mail: channy@umich.edu).

A. Ho is with the Dept. of Mechanical Engineering, University of Michigan, Ann Arbor, MI 48109 USA (e-mail: allho@umich.edu).

B. J. Martin is with the Dept. of Industrial \& Operations Eng., University of Michigan, Ann Arbor, MI 48109 USA (e-mail: martin@umich.edu).

K. H. Sienko is with the Depts. of Mechanical and Biomedical Engineering, University of Michigan, Ann Arbor, MI 48109 USA (phone: 734-647-8249; fax: 734-615-6647; e-mail: sienko@umich.edu). vibrations may provoke an avoidance response similar to bumping into an obstacle. Torso-based vibrotactile biofeedback systems incorporating repulsive cuing strategies have been shown to significantly reduce body sway in individuals with vestibular deficits [3-7], older adults [2, 8], and healthy young adults $[1,3,9]$ during quiet and perturbed stances [4-6].

However, repulsive cuing strategies may not be congruent with kinesthetic information from the stimulated tactile receptors. It has been shown that vibratory muscle stimulation modifies muscle spindle proprioceptive responses [10], generates illusions of movement [11-12], and leads to non-volitional balance-correcting responses [13-14]. In addition, extended periods of whole-body vibrations induce postural alterations [14]. Recently, we demonstrated that vibrotactile stimulations applied over the internal oblique and erector spinae muscles induce postural shifts in the direction of the applied stimulation in the absence of instruction [15-16]. Although simultaneous vibratory stimuli of two muscle groups results in the summation of postural responses [13], the effects of a combination of cutaneous stimuli applied to the torso remain unknown.

The objectives of the present study are to assess whether co-vibrotactile stimulations (a vibration stimulus applied simultaneously over two skin areas) around the torso has a summation effect on postural responses in the absence of instructions, and to determine if this summation has vectorial properties. The results from this study will be used to inform the design of displays for balance-related applications.

\section{METHODS}

\section{A. Instrumentation}

The experimental apparatus included a commercial six degree-of-freedom inertial measurement unit (IMU; Xsens Technologies, NL), a vibration control circuit, and six linear actuators subsequently referred to as tactors (C2, Engineering Acoustics Inc., USA). The IMU signals indicating angular displacements (static accuracy better than $0.5^{\circ}$ and a $0.05^{\circ}$ angular resolution) in the anterior-posterior $(\mathrm{A} / \mathrm{P})$ and medio-lateral $(\mathrm{M} / \mathrm{L})$ directions were sampled at $100 \mathrm{~Hz}$. The C2 tactors were placed on the skin over the left and right internal oblique, external oblique, and erector spinae muscles. Both the IMU and tactors were attached with Velcro to an elastic belt worn around the torso approximately at the level of the L4/L5 vertebrae. Tactors were activated by a $250 \mathrm{~Hz}$ sine wave to avoid the response of muscle spindles [17] and to 
maintain the stimulation within the one-to-one frequency response of fast-adapting cutaneous receptors [18]. The displacement amplitude was $200 \mu \mathrm{m}$ for all tactors [16].

\section{B. Subjects}

Four healthy young $(23.5 \pm 4.4$ yrs $)$ naïve subjects ( $2 \mathrm{M}, 2$ F) participated in this study. The University of Michigan Institutional Review Boards approved the experimental protocol, which conformed to the Helsinki Declaration. Informed consent was obtained from each subject prior to the start of the experiment.

\section{Experimental Protocol}

The subjects stood on a firm surface in two different stance conditions: normal stance, feet hip-width apart $(15 \mathrm{~cm}$ heel to heel distance) at a $15^{\circ}$ lateral rotation angle, and Romberg stance, feet together. The order of stance condition was randomized. Ear plugs eliminated environmental noise.

Each trial lasted $15 \mathrm{~s}$ and consisted of three consecutive periods: $5 \mathrm{~s}$ without vibration, $5 \mathrm{~s}$ with vibration, and $5 \mathrm{~s}$ without vibration. Vibrotactile stimulations were applied either simultaneously over the skin of two muscle groups (referred to as "co-vibration" conditions) or simultaneously over the skin of six muscle groups (referred to as "all locations" condition). Co-vibrations were a combination of two individual vibration locations as illustrated in Fig. 1: right and left internal oblique, right and left erector spinae, right internal oblique and right erector spinae, and left internal oblique and left erector spinae. Each subject was instructed to "stand in an upright position, keeping your knees extended and your arms at your side; breathe normally throughout the experiment (no sighing or breathing deeply during data collection)." Information regarding the duration and location of the vibrotactile stimulation was not provided.

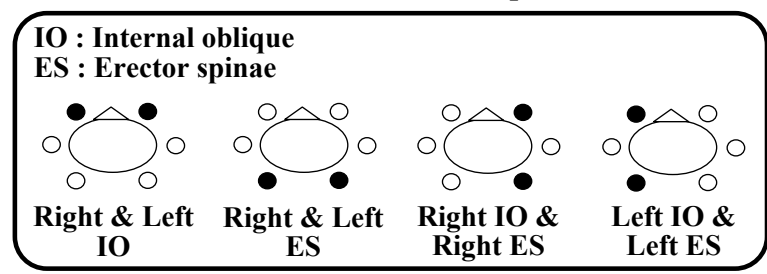

Figure 1. Four co-vibration conditions. Bird's-eye view drawings illustrate the location of the vibration stimulation.

\section{Data Analysis}

All IMU-captured postural sway signals were post-processed using MATLAB (The MathWorks, MA). For data analysis, the "pre-vibration" and "post-vibration" periods were defined as the $5 \mathrm{~s}$ preceding and following the vibrotactile stimulation (per-vibration) period, respectively. Two metrics were defined to quantify postural responses to vibrotactile stimulation: the postural shift vector (indicating the magnitude and direction of postural shift), and the root-mean-square (RMS) of the angular displacements of the body in the $\mathrm{A} / \mathrm{P}$ and $\mathrm{M} / \mathrm{L}$ directions.

To determine the magnitude and direction of postural responses between the consecutive periods of interest (pre-/per- and per-/post-vibration), 95\% confidence interval ellipses were fit to the 2D postural trajectories for each period. The center of each ellipse was used to calculate the 2D postural shift vector for each period. Detailed information regarding the computation of postural shift vectors was provided previously [16]. The magnitudes and directions of the postural shift vectors as well as the $\mathrm{A} / \mathrm{P}$ and $\mathrm{M} / \mathrm{L}$ RMS sway were computed for each period as a function of the stimulation condition and stance condition. Each metric was quantified by the average over two trials.

A preliminary ANOVA was conducted to determine the main effects of stance, location (four "co-vibration" and the "all locations" conditions), and period (pre-, per- and post-vibration) for each dependent variable (magnitude, direction, A/P RMS, and M/L RMS of postural sway). Hypotheses for the main effects of stance condition, location condition, and measurement period as well as their interactions were tested using an F-test. To determine which factors influenced the main and interaction effects, post-hoc tests (Tukey's HSD for multiple comparisons) were also conducted. The level of significance was set at $p<0.05$.

\section{RESULTS}

Postural Shift Magnitude and Direction: Fig. 2 presents the postural shift vectors during and after vibration as a function of the vibration condition for normal stance. The ANOVA applied to the postural shift vectors (i.e., postural shift magnitude and direction) indicated that the main effects of the location (Normal: $F(4,45)=2.79, p=0.037$, Romberg: $F(4,45)=3.09, p=0.025)$, period (Normal: $F(2,45)=19.91, p$ $<0.001$, Romberg: $F(2,45)=5.14, p=0.011)$, and location $\times$ period interactions (Normal: $F(8,45)=2.79, p=0.013$, Romberg: $F(8,45)=3.94, p=0.001)$ were significant. Post-hoc analysis showed that the magnitude of the postural shift vectors was significantly greater during, than before or after, the vibration period for both stance conditions $(p<$ 0.01 ) when co-vibration was applied over the right and left internal oblique and over the right and left erector spinae muscles. For these two co-vibration conditions, the average postural shift vector induced by vibration was not significantly different between the two stance conditions ( $p>$ 0.05 ). The postural shift occurred in the anterior direction when co-vibration was applied over the right and left internal oblique muscles, while the shift occurred in the posterior direction when co-vibration was applied over the right and left erector spinae muscles. Upon cessation of vibration, the direction of postural shift vectors was similar to that of postural shift vectors during vibration. However, the magnitudes and directions of the postural shift vectors did not significantly change when vibration was applied to all locations, or when co-vibration was applied to the right internal oblique and right erector spinae muscles and to the left internal oblique and left erector spinae muscles. 

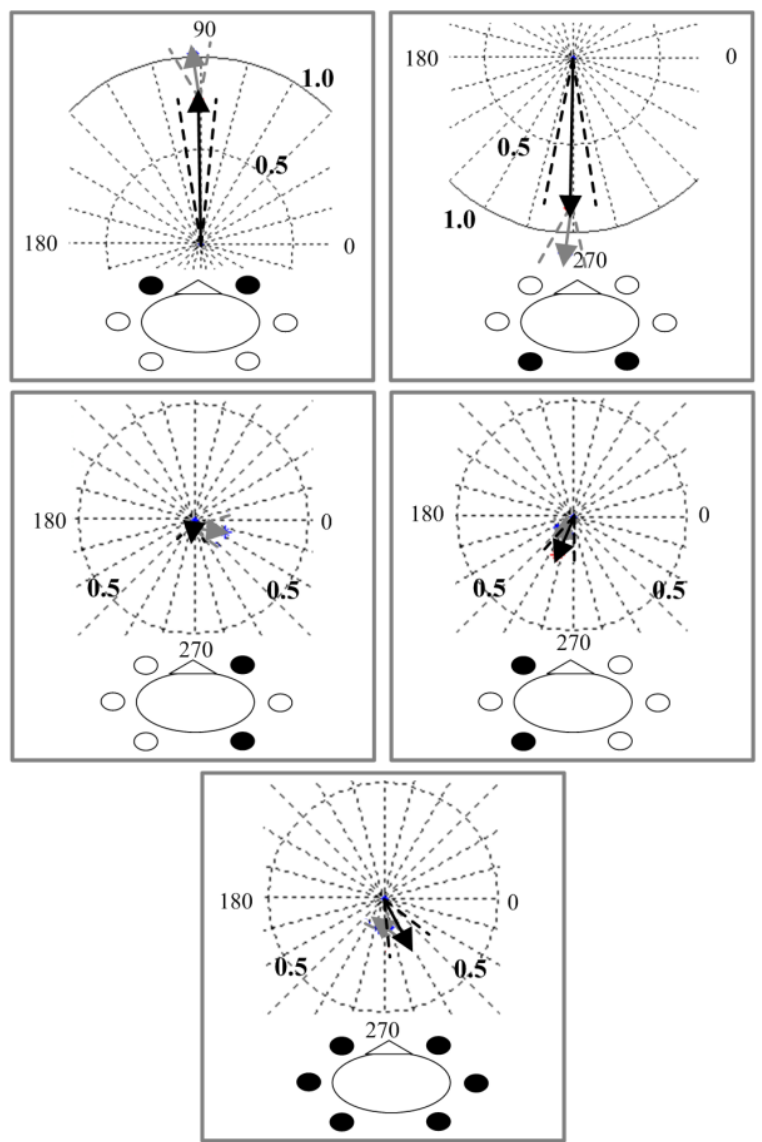

Figure 2. Average postural shift vectors during and after vibration as a function of the vibration condition. (a) Right and left internal oblique. (b) Right and left erector spinae. (c) Right internal oblique and right erector spinae. (d) Left internal oblique and left erector spinae. (e) All locations. Black and grey vectors represent postural shifts for per- and post-vibration periods, respectively. Dashed lines indicate standard error of the corresponding mean vector.

The average latency of vibration-induced postural shifts for both stance conditions when co-vibration was applied over internal oblique and erector spinae muscles was approximately $900 \mathrm{~ms}$ after the onset of vibration. This latency was not statistically different $(p>0.05)$ for the co-vibration of the right and left internal oblique muscles and of the right and left erector spinae muscles for both stance conditions.

RMS sway: Fig. 3 shows the A/P RMS sway during vibration as a function of the location condition and stance condition. The ANOVA applied to A/P RMS sway indicated that the main effects of the location (Normal: $F(4,45)=13.14$, $p<0.001$, Romberg: $F(4,45)=10.33, p<0.001)$, period (Normal: $F(2,45)=34.69, p<0.001$, Romberg: $F(2,45)=$ $33.86, p<0.001$ ), and location $\times$ period interactions (Normal: $F(8,45)=8.18, p<0.001$, Romberg: $F(2,45)=8.61, p<0.001)$ were significant. However, ANOVA applied to M/L RMS sway indicated neither significant main or interaction significant effects. Post-hoc analysis showed that the A/P RMS sway was significantly greater $(p<0.001)$ during, than before or after, the vibration period when co-vibration was applied over the right and left internal oblique muscles and the right and left erector spinae muscles, regardless of the (a)

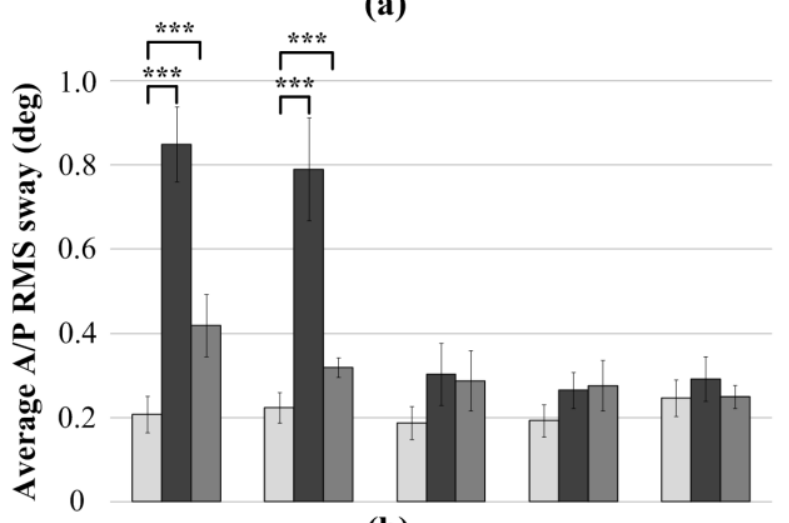

(b)

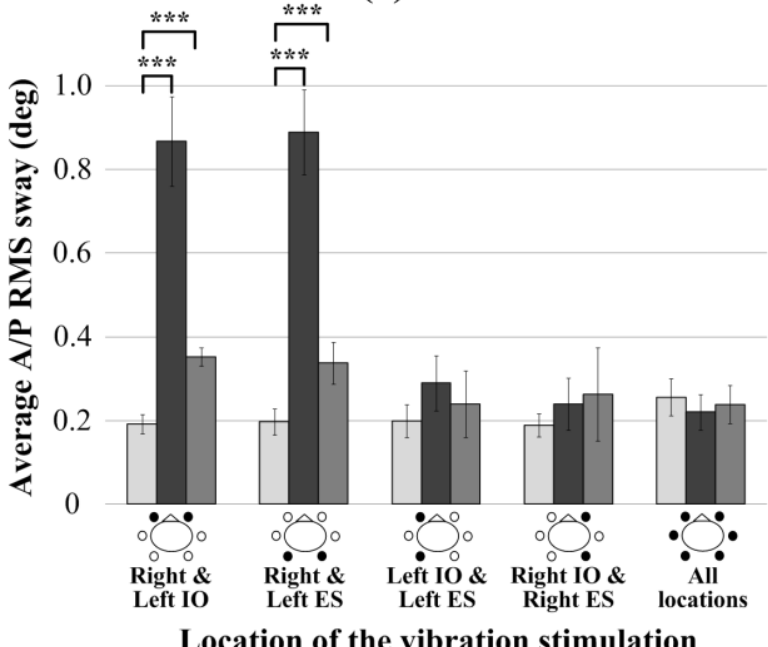

Figure 3. Average A/P RMS sway as a function of the location of the vibration stimulation. (a) Normal stance. (b) Romberg stance. Light, dark, and intermediate grey bars represent the pre-, per-, and post-vibration periods, respectively. Error bars indicate standard error of the mean $(* * * p<0.0001)$. Bird's-eye view drawings illustrate the location of the vibration stimulation. IO and ES indicate the internal oblique and elector spinae muscles, respectively.

stance condition. For these two co-vibration conditions, the average A/P RMS sway induced by vibration was not significantly different between the two stance conditions $(p>$ $0.05)$. However, changes in the $\mathrm{A} / \mathrm{P}$ sway were negligible when vibration was applied over the combined right internal oblique with right erector spinae muscles, the combined left internal oblique with left erector spinae muscles, and all locations, regardless of the stance.

\section{Discussion}

Co-vibration of homologous skin areas (symmetry relative to the mid sagittal plane) over torso prime mover muscles induced significant postural shifts in both stance conditions tested. The $900 \mathrm{~ms}$ latency of these responses is not compatible with a cutaneous reflex contribution [19]. Furthermore, in each condition, the direction of the shift corresponds to the vector summation of the directional shifts induced by vibrotactile stimulation applied individually to the respective skin area of the tested pairs observed in a previous study [15-16]. Hence, the present results confirm that changes 
in posture resulting from vibrotactile stimulation are non-volitional, and reflect a behavioral response associated with a vibration-induced alteration of cutaneous information similar to the responses induced by alteration of muscle proprioception (e.g., [13]). The vector summation, which is similar to the one observed with muscle tendon co-vibration $[13,20]$, strongly supports that cutaneous receptors located in the skin covering torso prime mover muscles contribute to proprioception and body representation in space. In addition, the directional coding reflects the properties of population afferents issued from the joint skin areas [21] which encode orientation of human joint movements. Taken together, these results suggest that cutaneous proprioception may be at least a property of human joint skin areas.

Co-vibration applied to the skin over either the right internal oblique and right erector spinae muscles or the left internal oblique and left erector spinae muscles did not induce a significant shift regardless of the stance condition. This absence of effect does not contradict a summation of effect that would induce a lateral shift. Indeed, vibration applied individually over the external oblique muscles did not induce a postural shift [15-16]. Furthermore, since postural stability is usually greater in the $\mathrm{M} / \mathrm{L}$ than $\mathrm{A} / \mathrm{P}$ direction during standing [14], a small vibration-induced postural change is less likely to induce a compensatory postural response.

Both normal and Romberg stances were chosen to verify whether or not the effects of co-vibration were consistent between the stances. Kirby et al. [22] did not find a significant difference in $\mathrm{A} / \mathrm{P}$ travel between feet together and $15 \mathrm{~cm}$ apart, or feet straight and toes out at a $25^{\circ}$ or $45^{\circ}$ angle. This study also found an absence of difference.

In summary, these preliminary results show that in the absence of instructions, stimuli combinations applied to the skin over torso prime mover muscles result in a vector summation of postural responses obtained with individual stimulations. This finding may have implications for improving the performance of vibrotactile biofeedback devices. Individual or combined cutaneous stimulations may be used to effectively encode the direction of a postural adjustment. Since the involuntary response to these stimulations is in a direction corresponding to their location of application, the instruction given to individuals should be to "move toward the vibration" to increase the compatibility between the non-volitional and volitional postural adjustments. We suggest that this compatibility may contribute to both a reduction of response latency and the efficiency of the biofeedback method.

\section{ACKNOWLEDGMENT}

We would like to acknowledge the University of Michigan Center for Statistical Consultation and Research.

\section{REFERENCES}

[1] J. F. Janssen, L. Verhoeff, G. C. Horlings, and H. J. Allum, "Directional effects of biofeedback on trunk sway during gait tasks in healthy young subjects," Gait Posture vol. 29, no. 4, pp. 575-581, Jun. 2009.
[2] A. Karen, "Vibrotactile Postural Control in Patients That Have Sit-To-Stand Balance Deficit \& Fall," Ph.D. dissertation, Physical Therapy, Nova Southeastern University, Davie, 2010.

[3] B. C. Lee, J. Kim, S. Chen, and K. H. Sienko, "Cell phone based balance trainer," J Neuroengineering Rehabil, vol. 9, no. 10, Feb. 2012.

[4] K. H. Sienko, M. D. Balkwill, L. I. Oddsson, and C. Wall, "Effects of multi-directional vibrotactile feedback on vestibular-deficient postural performance during continuous multi-directional support surface perturbations," J Vestib Res, vol. 18, no. 5-6, pp. 273-285, Jan. 2008.

[5] K. H. Sienko, M. D. Balkwill, and C. Wall, "Biofeedback improves postural control recovery from multi-axis discrete perturbations," $J$ Neuroengineering Rehabil, In press.

[6] K. H. Sienko, V. V. Vichare, M. D. Balkwill, and C. Wall, "Assessment of vibrotactile feedback on postural stability during pseudorandom multidirectional platform motion," IEEE Trans Biomed Eng, vol. 57, no. 4, pp. 944-952, Apr. 2010.

[7] C. Wall and E. Kentala, "Control of sway using vibrotactile feedback of body tilt in patients with moderate and severe postural control deficits," J Vestib Res, vol. 15, no. 5-6, pp. 313-325, Apr. 2005.

[8] S. Haggerty, L. T. Jiang, A. Galecki, and K. H. Sienko, "Effects of biofeedback on secondary-task response time and postural stability in older adults," Gait Posture, vol. 35, no. 4, pp. 523-528, Apr. 2012.

[9] C. Wall, M. S. Weinberg, P. B. Schmidt, and D. E. Krebs, "Balance prosthesis based on micromechanical sensors using vibrotactile feedback of tilt," IEEE Trans Biomed Eng, vol. 48, no. 10, pp. 1153-1161, Oct. 2001.

[10] J. P. Roll, J. P. Vedel, and E. Ribot, "Alteration of proprioceptive messages induced by tendon vibration in man: a microneurographic study," Exp Brain Res, vol. 76, no. 1, pp. 123-222, 1989.

[11] G. M. Goodwin, D. I. McCloskey, and P. B. Matthews, "The contribution of muscle afferents to kinaesthesia shown by vibration induced illusions of movement and by the effects of paralysing joint afferents," Brain, vol. 95, no. 4, pp. 705-748, 1972.

[12] J. R. Lackner and M. S. Levine, "Changes in apparent body orientation and sensory localization induced by vibration of postural muscles: vibratory myesthetic illusions," Aviat Space Environ Med, vol. 50, no. 4, pp. 346-354, Apr. 1979.

[13] A. Kavounoudias, R. Roll, and J. P. Roll, "Foot sole and ankle muscle inputs contribute jointly to human erect posture regulation," J Physiol, vol. 532, no. Pt 3, pp. 869-878, May. 2001.

[14] B. J. Martin, G. M. Gauthier, J. P. Roll, M. Hugon, and F. Harlay, "Effects of whole-body vibrations on standing posture in man," Aviat Space Environ Med, vol. 51, no. 1, pp. 778-787, Aug. 1980.

[15] B. C. Lee, B. J. Martin, and K. H. Sienko, "Directional postural responses induced by vibrotactile stimulations applied to the torso," unpublished.

[16] B. C. Lee, B. J. Martin, and K. H. Sienko, "Comparison of non-volitional postural responses induced by two types of torso based vibrotactile stimulations," in IEEE Haptics Symposium, Vancouver, Canada, 2012 pp. 195-198.

[17] D. Burke, K. E. Hagbarth, L. Lofstedt, and B. G. Wallin, "The responses of human muscle spindle endings to vibration during isometric contraction," J Physiol, vol. 261, no. 3, pp. 695-711, Oct. 1976.

[18] E. Ribot-Ciscar, J. P. Vedel, and J. P. Roll, "Vibration sensitivity of slowly and rapidly adapting cutaneous mechanoreceptors in the human foot and leg," Neurosci Lett, vol. 104, no. 1-2, pp. 130-135, Sep. 1989.

[19] E. Kugelberg and K. E. Hagbarth, "Spinal mechanism of the abdominal and erector spinae skin reflexes," Brain, vol. 81, no. 3, pp. 290-304, Sep. 1958.

[20] P. Romaiguère, J.-L. Anton, M. Roth, L. Casini, and J.-P. Roll, "Motor and parietal cortical areas both underlie kinaesthesia," Brain Res Cogn Brain Res, vol. 16, no. 1, pp. 74-82, Mar. 2003.

[21] J. M. Aimonetti, V. Hospod, J. P. Roll, and E. Ribot-Ciscar, "Cutaneous afferents provide a neuronal population vector that encodes the orientation of human ankle movements.," J Physiol, vol. 580, no. 2, pp. 649-658, Apr. 2007.

[22] R. L. Kirby, N. A. Price, and D. A. MacLeod, "The influence of foot position on standing balance," J Biomech, vol. 20, no. 4, pp. 423-427, 1987. 